\title{
Sampling frequency impact on False Lock of High Order BOC Signals in Open-Loop Processing
}

\author{
Adrià Gusi*, Pau Closas* and J.A. Garcia-Molina ${ }^{\dagger}$ \\ *Centre Tecnològic de Telecomunicacions de Catalunya (CTTC) \\ Spain \\ $\dagger$ ESA ESTEC/TEC-ETN, and HE SPACE \\ The Netherlands
}

\begin{abstract}
The modernized GNSS systems introduced the idea of BOC signals, which provide better tracking accuracy than BPSK signals at the cost of having side lobes in the autocorrelation function. In closed-loop processing, or tracking, the presence of side lobes can origin false locks, thus making the ranging estimate to be biased by several meters. The overall position, velocity and time solution can then be severely distorted in that situation. The false lock in high order BOC signals is a well known problem and several state-of-the-art techniques address it. This article evaluates the problem from an open-loop perspective. In particular, the impact of the sampling frequency on the probability of false lock is examined.
\end{abstract}

\section{INTRODUCTION}

The current Global Navigation Satellite Systems (GNSS) use Binary Offset Carrier (BOC) modulated signals. These signals have a narrower main lobe of the autocorrelation function (ACF) with respect to the classic Binary Phase Shift Keying (BPSK) modulated signals, and hence they offer better tracking accuracy [1]. However, the drawback comes in the side lobes appearing in the ACF, that can origin false locks in the tracking process. In the case of high-order BOC signals, the problem becomes more evident as there is a large number of side lobes and their amplitude can be close to the main lobe. Also, the lobes adjacent to the main lobe incur a ranging bias of only a few meters, and hence, the false lock can be easily overlooked. Moreover, in harsh propagation conditions the amplitude of the side lobes can increase and even overtake the main lobe. This can be the case of a mobile user in the urban canyon where both rich multipath and shadow fading are present.

The false lock issue has been widely addressed in the literature in terms of tracking or closed-loop processing. The problem can be avoided if the equivalent BPSK envelope is tracked, at the cost of losing the higher accuracy of the BOC signal. Other techniques aim at tracking the main lobe of the original BOC signal. In general, they are designed in a way to detect and subsequently correct a false lock. Such state-of-the art techniques are Bump Jumping [2], Double Estimator[3], Code-Subcarrier Smoothing [4], or the Astrium correlator [5]. This techniques require, in general, a low number of correlators.

Another interesting approach to deal with the false lock phenomena is open-loop processing. In an open-loop approach, the signal is processed in batches and a set of parameters are estimated independently from each data batch. The idea is equivalent to the acquisition step of a GNSS receiver. Some $a$ priori information regarding the parameters can be taken into account to reduce the search complexity, which is comparable with reaquisiton step. In open-loop processing there is a larger number of observable correlators with respect to closed-loop processing. Therefore, the main lobe and also some or all the side lobes can be observed. One can exploit this information to determine the TOA of the main lobe and avoid false locking.

Open-loop processing techniques appear as a promising approach to deal with the false lock phenomena. Some novel techniques target this issue with a multi-correlator approach [6], [7]. The use of an increased number of correlators contributes to a global view of the $\mathrm{ACF}$, including the main lobe and some or all of its side lobes. This information can be employed to properly determine the position of the main lobe and distinguish it from sidelobes. In [8] the false lock probability is evaluated assuming that the ACF is sampled in a way enabling to distinguish the different side lobes.

In this contribution we study the impact of the sampling frequency $f_{s}$ on the probability of false lock of high order BOC signals. We consider that the data is processed in OpenLoop. Hence, the samples are arbitrary taken within a subsample range equal to the sampling period $T_{s}=1 / f_{s}$. This means that the main peak might not be sampled at its largest amplitude, whereas, for instance, a secondary lobe could be sampled at a larger amplitude. The conventional approach to estimate the time delay is to find the sample that maximizes the cross-correlation between the received signal and the local replica. Such estimator can therefore suffer from false locking due to the sampling frequency. An alternative method capitalized on the maximum likelihood (ML) of the correlated samples is proposed.

The remainder of the paper is organized as follows. Section II presents the mathematical model considered throughout the article. Section III introduces the false lock problem due to the sampling frequency that takes place with the conventional approach. In Section IV an alternative approach dealing with the false lock is proposed. In we compare both approaches by means of simulations. Finally, Section VI concludes the paper. 


\section{SYSTEM MODEL}

We assume here that carrier phase and Doppler-shift are perfectly wiped off. The received GNSS signal through a lineof-sight signal (LOSS) propagation channel can be expressed in baseband as

$$
r(t)=a s(t-\tau)+w(t),
$$

where $s(t)$ is the transmitted signal with energy $E_{s}, a$ is the received complex amplitude, $\tau$ is the time of arrival (TOA), $\phi$ is the phase of the received signal, and $w(t)$ is the complex additive white Gaussian noise (AWGN) with variance $\sigma_{w}^{2}$. The complex amplitude can be expressed as $a=\alpha e^{j \phi}$, with $\alpha$ and $\phi$ being the amplitude and phase, respectively. The time delay $\tau$ is considered to be a deterministic unknown parameter.

A $\operatorname{BOC}(m, n)$ signal is obtained after superimposing a square wave subcarrier to the spreading code of a BPSK signal [9]. The square wave subcarrier has frequency $f_{s c}=$ $\frac{1}{2 T_{s c}}=m f_{g}$, where $T_{s c}$ is half-period of a square wave and $f_{g}=1.023 \mathrm{MHz}$. The chip rate of the underlying BPSK modulation is given by $f_{c}=\frac{1}{T_{c}}=n f_{g}$, where $T_{c}$ is the chip period. The resulting signal can be written as

$$
s_{B O C}(t)=s_{B P S K}(t) \operatorname{sgn}\left(\sin \left(\pi t / T_{s c}+\psi\right)\right)
$$

where $\psi$ is an adjustable phase angle. The phase is typically chosen to generate a sine phased signal $(\psi=0)$ or a cosine phased signal $(\psi=\pi / 2)$. The BPSK signal can be expressed as

$$
s_{B P S K}(t)=\sqrt{2 P_{s}} a_{k} g\left(t-k T_{c}\right),
$$

where $P_{s}$ is the transmitted signal power, $a_{k} \in\{-1,1\}$ are the pseudo random noise (PRN) sequence values, and $g(t)$ is the energy-normalized chip shaping pulse.

The received signal is correlated with a local replica $s(t-\theta)$, where $\theta$ is the unknown parameter associated with $\tau$. The obtained cross-correlation is as follows

$$
R_{s r}(\theta)=\int_{-\infty}^{+\infty} s(t-\theta) r(t) d t=\alpha R_{s}(\theta-\tau)+n(\theta)
$$

with

$$
R_{s}\left(\theta-\theta^{\prime}\right)=\int_{-\infty}^{+\infty} s(t-\theta) s\left(t-\theta^{\prime}\right) d t
$$

being the autocorrelation of $s(t)$, and $n(\theta)=\int_{-\infty}^{+\infty} s(t-$ $\theta) w(t) d t$ is the correlation of $w(t)$ with $s(t-\theta)$, which results in colored zero-mean Gaussian noise. The covariance of $n(\theta)$ is found as

$$
\operatorname{Cov}\left(n(\theta), n\left(\theta^{\prime}\right)\right)=\mathbb{E}\left\{n(\theta) n\left(\theta^{\prime}\right)\right\}=\sigma_{w}^{2} R_{s}\left(\theta-\theta^{\prime}\right) .
$$

The received signal is converted to discrete time at a sampling frequency $f_{s}=\frac{1}{T_{s}}$ where $T_{s}$ is the sampling period. The discrete cross-correlation signal can be written as

$$
R_{s r}[k]=R_{s r}\left(k T_{s}\right)=\alpha R_{s}[k-\tau]+n[k]
$$

The event of false lock needs to be defined in order to proceed with the analysis. Let us assume that an estimate of $\tau$ is obtained and denoted as $\hat{\tau}$. The estimation error can be

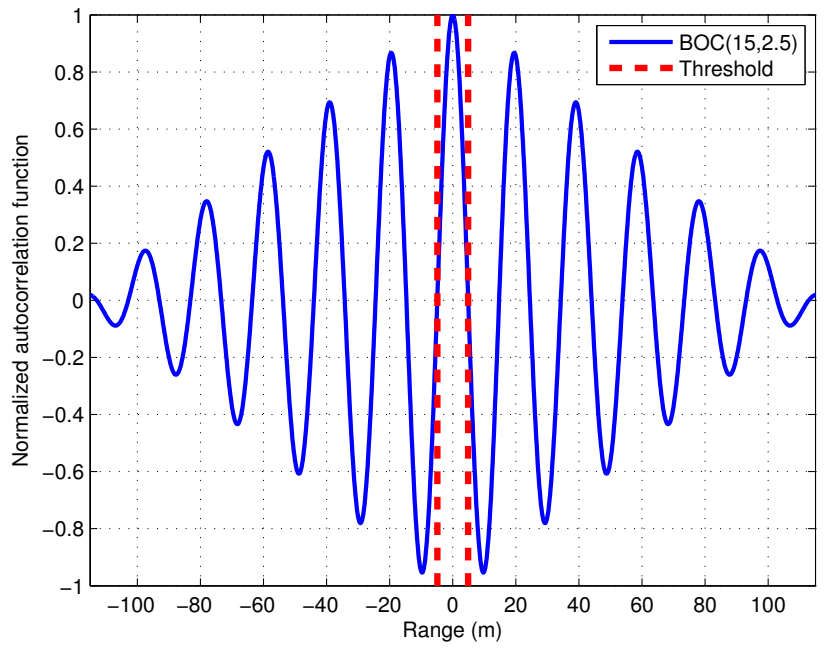

Fig. 1. Filtered autocorrelation function of a $\operatorname{BOC} \cos (15,2.5)$ signal.

then written as $\epsilon=\hat{\tau}-\tau$. A false lock occurs when the estimation error is above a certain threshold. In this article we set the lock region limit at the middle point between the main peak and the closest side lobe. The threshold is found to be $a=T_{s c} / 2$ for all BOC modulations. The values of $\hat{\tau}$ that satisfy $|\hat{\tau}-\tau|<T_{s c} / 2$ belong to the lock region, i.e., the region around the main lobe [8].

The false lock threshold is illustrated in Figure 1. The ACF of a cosine phased $\mathrm{BOC}(15,2.5)$ is shown. The signal was filtered with a $40 \mathrm{MHz}$ bandwidth low-pass filter, to emulate the front-end filter of a GNSS receiver. Note that the lock region is set 5 meters away from the main peak, and that locking on the first side lobe represents a systematic ranging error of roughly 10 meters.

\section{FALSE LOCK INDUCED BY THE SAMPLING PROCESS}

The conventional approach to estimate the time delay is to find the value of $\theta$ that maximizes the cross-correlation function in (4). This method is obtained after deriving the ML estimator for the signal model in (1) and it can be expressed as

$$
\hat{\tau}=\arg \max _{\theta}\left|R_{s r}(\theta)\right|,
$$

where the absolute value of $R_{s r}(\theta)$ is taken since the phase $\phi$ is unknown. Note that in continuous time we can be as accurate as needed in the search of $\theta$. In discrete-time however, we perform the following maximization

$$
\hat{k}=\arg \max _{k}\left|R_{s r}[k]\right| .
$$

The sample that maximizes the function is selected, and the associated time delay estimate is computed as $\hat{\tau}=\hat{k} T_{s}$. This value has a resolution equal to the sampling period $T_{s}=\frac{1}{f_{s}}$. Therefore, a finer estimation of the time delay needs to be obtained. In tracking, the delay-locked loop (DLL) discriminator can efficiently set the tracking around the peak 
of the ACF. In open-loop approaches, one can interpolate a few samples around the argument of the maximum sample.

Nevertheless, the estimate $\hat{k}$ can be sometimes found outside the lock region defined in Section II. The use of this initial value would incur on a false lock in the subsequent step of tracking in a conventional GNSS receiver. Also, in the case of open-loop processing, the use of classic interpolation would yield a false lock.

\section{POST-CORRELATION ML ESTIMATOR}

One solution for the sampling induced false lock problem is to perform a ML estimation of the time delay with the postcorrelation samples. One can view this as finding the model that fits better with the data, taking into account the distribution of the data. The concept was proposed in [6], [7] to solve the problem of ambiguous estimation. In the following, we present the mathematical derivation of the proposed estimator.

Let us assume that $K$ samples are taken from the crosscorrelation function in (7). The samples are denoted $k_{n}$, with $n=1, \ldots, K$. Let us express the vector of $K$ cross-correlation samples as

$$
\mathbf{x}=\left(x_{1} \cdots x_{K}\right)^{\top}=\left(R_{s r}\left[k_{1}\right] \cdots R_{s r}\left[k_{K}\right]\right)^{\top} .
$$

The negative $\log$-likelihood function of $\tau$ and $\alpha$, neglecting the constant terms, is given by

$$
\Delta(\tau, \alpha)=(\mathbf{x}-a \mathbf{s}(\tau))^{H} \mathbf{\Sigma}^{-1}(\mathbf{x}-a \mathbf{s}(\tau)),
$$

where

$$
\left.\boldsymbol{s}(\tau)=R_{s}\left[k_{1}-\tau\right] \cdots R_{s}\left[k_{N}-\tau\right]\right)^{\top}
$$

is the vector of autocorrelation samples, and

$$
\boldsymbol{\Sigma}=\mathbb{E}\left\{\mathbf{x x}^{\top}\right\}=\sigma_{w}^{2}\left[R_{s}\left[k_{n}-k_{n^{\prime}}\right]\right]_{n, n^{\prime}=n_{1}, \cdots, n_{N}}
$$

is the covariance matrix. Note that the covariance matrix can be constructed upon evaluating the autocorrelation function in (5), and hence it does not need to be estimated. The ML estimate of the complex received amplitude $a$ is given by

$$
\hat{a}=\left(\boldsymbol{s}(\tau)^{\top} \boldsymbol{\Sigma}^{-1} \boldsymbol{s}(\tau)\right)^{-1} \boldsymbol{s}(\tau)^{\top} \boldsymbol{\Sigma}^{-1} \mathbf{x}
$$

The estimate of $a$ can be inserted in the log-likelihood expression (11). The ML estimate of $\tau$ is then given by the solution of the following non-linear minimization problem

$$
\hat{\tau}=\arg \min _{\tau}(\mathbf{x}-\hat{a} \mathbf{s}(\tau))^{H} \mathbf{\Sigma}^{-1}(\mathbf{x}-\hat{a} \mathbf{s}(\tau)) .
$$

Note that the derived estimator directly obtains a fine estimate of $\tau$, whereas the conventional estimator in (9) obtains an estimate within the sampling period resolution. This is because the local replica used in (15) can be evaluated at any point and is not restricted to the sampling of the ACF.

\section{NUMERICAL RESULTS}

In this section we compare the performance of the conventional estimation approach explained in Section III with performance of the post-correlation ML estimator introduced in Section IV.

In order to simulate the impact of the sampling frequency on the false locking, we have to take into account that the ACF does not always exhibit a sample precisely on the main peak. The samples are in fact distributed in a uniform distribution with interval length of a sampling period around the main peak. One way to simulate this is to generate values of $\tau$ such that $\tau=\gamma+\delta$, where $\gamma$ is a deterministic parameter, and $\delta$ is a random variable $\delta \sim \mathcal{U}\left(-\frac{T_{s}}{2}, \frac{T_{s}}{2}\right)$, accounting for the subsample delay. Note that another way to model the sub-sample delay is to introduce the delay in the generation of the local replica. Either way, the outcome is equivalent. if we apply the sub-sample delay, we have that the estimate of $k$ is

$$
\hat{k}=\arg \max _{k}\left|R_{s}[k-\gamma-\delta]+n[k]\right| .
$$

The above estimator is the one employed for the conventional approach. In the case of the ML estimator, the estimator in (15) is considered, and the time delay $\tau$ is generated in the same way as for the conventional approach. The samples of the $\mathrm{ACF}$ are taken in the range $\left[-T_{c}, T_{c}\right]$, meaning that the whole ACF is sampled. Note that the number of samples depends on the sampling frequency being evaluated.

Let us start with the results of the conventional approach. In Figure 2 we show the false lock probability with respect to the sampling frequency $f_{s}$. A filtered cosine-phased BOC $(15,2.5)$ signal and the estimator in (16) are considered. The simulation has been conducted without noise and, therefore, the observed false lock probability is due to the sampling frequency only. The false lock probability exhibits a trend to decrease as the sampling frequency increases, and for some particular values of $f_{s}$ the probability of false lock drops to zero. These values are around $f_{s}=61 \mathrm{MHz}, f_{s}=92 \mathrm{MHz}, f_{s}=124$ and $f s=144 \mathrm{MHz}$. These frequencies are multiple of the subcarrier frequency, $f_{s c}=15 f_{g}=15.345 \mathrm{MHz}$. When the $\mathrm{ACF}$ is sampled at these frequencies, we are ensuring that delay between two adjacent peaks of the ACF is divisible by the sampling period and hence, that if one sample is taken around one peak, the other peaks will be sampled as well.

It is to be noticed that a high sampling frequency does not guarantee false lock free estimates with the conventional approach. For instance, for $f_{s}=100 \mathrm{MHz}$ the probability of false lock is roughly 0.3 . It is only above $f_{s}=145 \mathrm{MHz}$ that false locks locks stop taking place.

For the sake of clarity, Figure 3 illustrates the ACF of a filtered BOC $(15,2.5)$ signal sampled at $f_{s}=61 \mathrm{Mhz}$, together with its respective continuous signal. It can be noticed that even if the samples are not taken at the maximum of the lobes, the global maximum is still taken at the region of the main peak. The reason is that the sampling period is a multiple of the distance between adjacent lobes, and hence, the samples are 


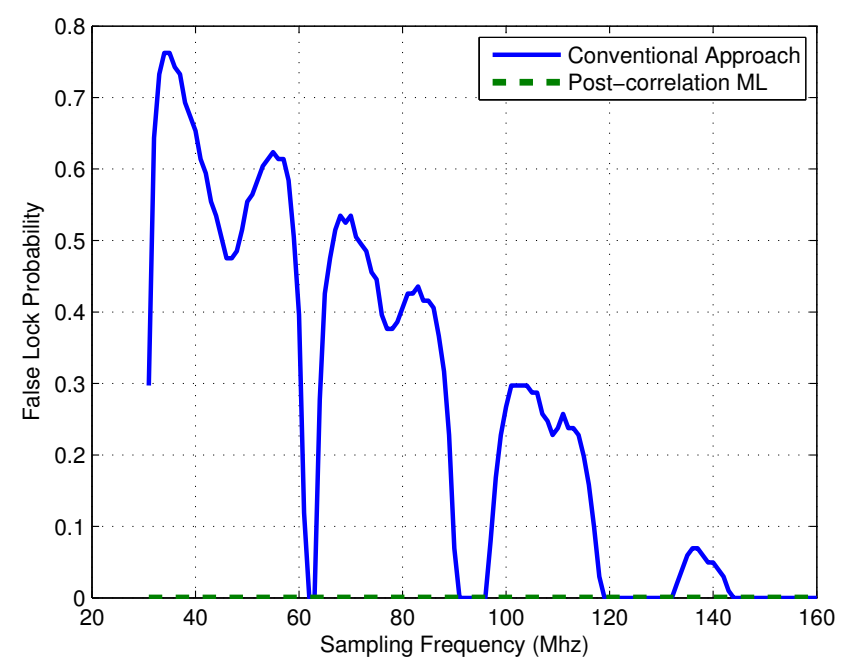

Fig. 2. False lock probability with respect to the sampling frequency for a filtered BOC $(15,2.5)$ signal.

taken in a proportional way with respect to the original amplitude. Also, the shape of the absolute value of the ACF can be observed in the figure. The absolute value operator halves the period of the ACF, and hence the effective frequency of ACF to be doubled. Thus, in practice, the sampling frequency needs to be multiple of twice the subcarrier frequency $2 f_{s c}=30.69$ MHz. Moreover the sampling frequency $f_{s}=30.69 \mathrm{MHz}$ is not suitable as it does not satisfy the Nyquist criterion. Therefore, the sampling frequencies that minimize the false lock probability are found as $f_{s}=\kappa 30.69 \mathrm{MHz}$ for $\kappa \in \mathbb{N}_{>1}$. In Figure 4 an example of false lock induced by the sampling frequency is shown. In this case, the received signal is sampled at $50 \mathrm{MHz}$. It can be seen that the sample with the maximum value corresponds to one of the side lobes next to the main one, incurring on a ranging bias of 10 meters.

If we consider that there is no front-end filter, the periodic nulls of the sampling frequency become more apparent. In Figure 5 we plot the false lock probability for a cosinephased BOC $(15,2.5)$. If the signal is not filtered the false lock probability is higher and is still relevant at higher values of sampling frequency with respect to the filtered signals. This is due to the sharper shape of the former.

The performance of the ML estimator of $\tau$ from the correlated samples (cf. Section IV) is also assessed. In Figure 2 the false lock probability was shown for both approaches. Recall that for this scenario no noise is generated. It appears from the figure that the post-correlation ML approach can reduce to zero the false locking for all the sampling frequencies evaluated. The result is particularly interesting at low sampling frequencies, where the original false lock probability can be around 0.7 and drops to zero after the ML estimation. We can use Figure 4 to better understand the underlying idea of the proposed ML based estimator. One can interpret the Original $\mathrm{ACF}$ curve depicted in the figure as a local replica of the $\mathrm{ACF}$

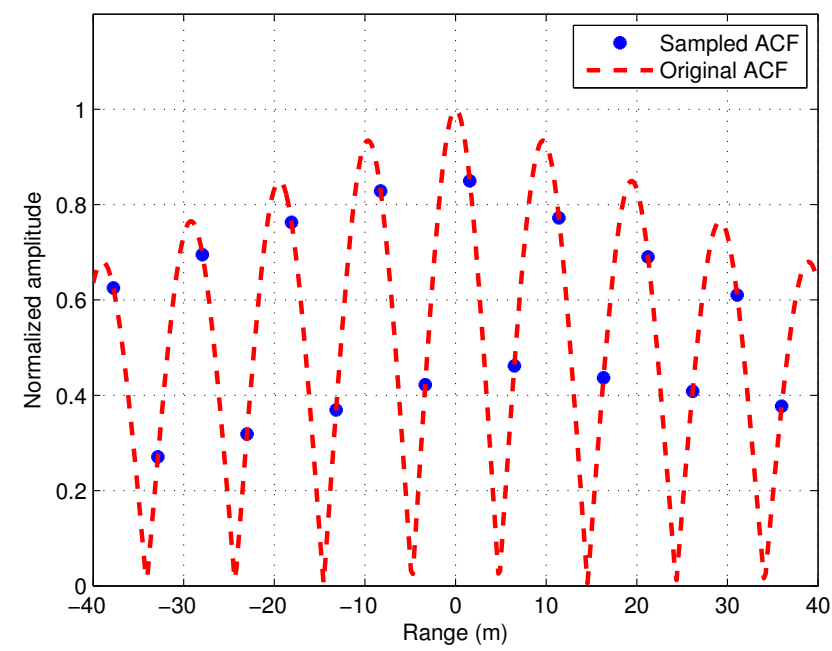

Fig. 3. ACF sampled at $61 \mathrm{Mhz}$

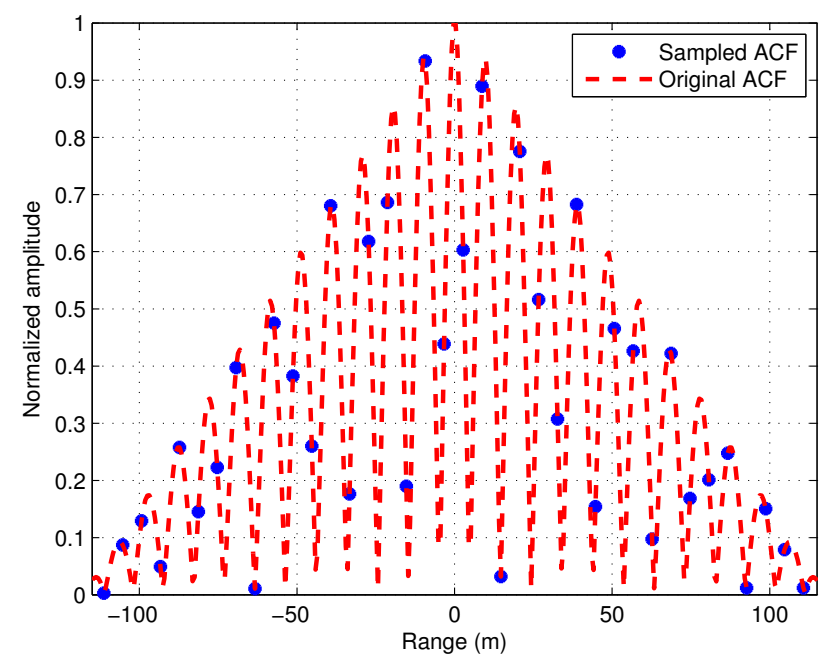

Fig. 4. ACF sampled at $50 \mathrm{Mhz}$

that is used as a template to fit the data. For a particular value of $\theta$, the cost function in (15) sees its minimum. The search on $\theta$ would represent shifting the replica model for higher or lower range values.

In Figure 6 we assess the performance of both approaches with respect to $C / N_{0}$ values, which can be computed from the model introduced in Section II as $C / N_{0}=\frac{\alpha^{2} E_{s}}{\sigma_{w}^{2} T_{0}}$, where $C$ is the received signal power, and $T_{0}$ is the integration time. A filtered BOC $(15,2.5)$ signal is considered, with sampling frequency set to $f_{s}=50 \mathrm{MHz}$. The conventional approach and the proposed estimator exhibit similar performances at low $C / N_{0}$. However, at mid and high $C / N_{0}$ the post-correlation ML estimator outperforms the conventional estimator. Also note that the conventional approach converges to a minimum false lock probability value of approximately 0.55 . This result 


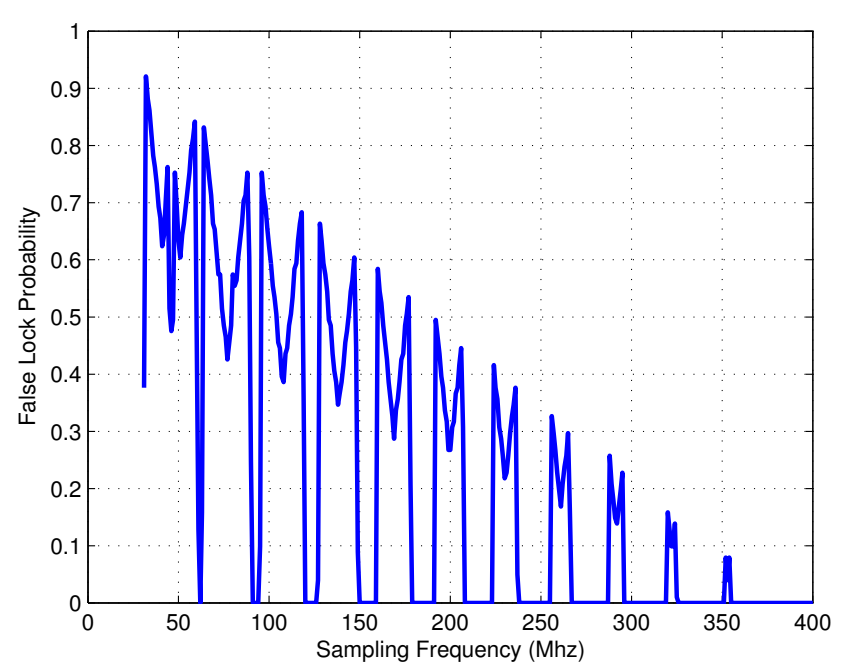

Fig. 5. False lock probability with respect to the sampling frequency for a BOC $(15,2.5)$ signal.

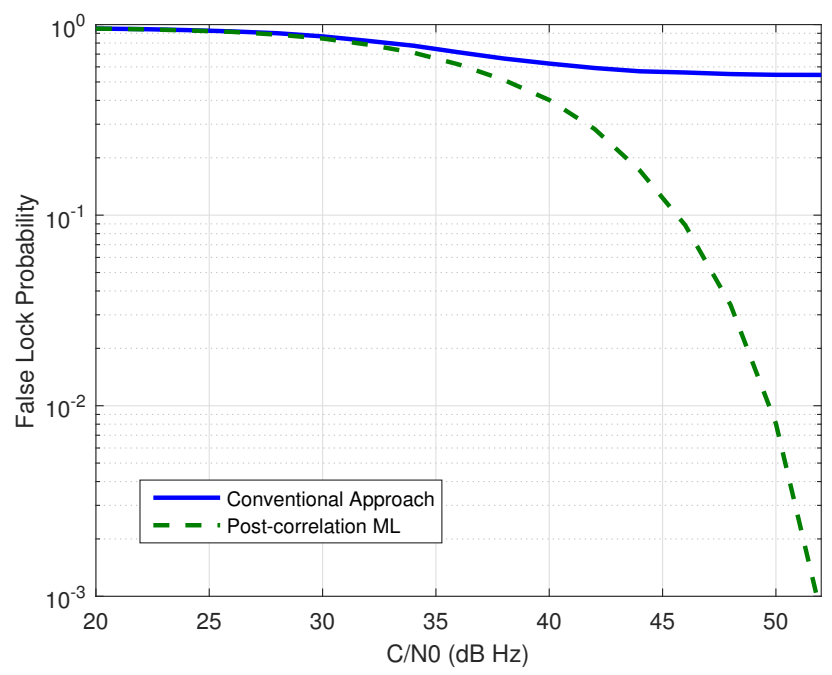

Fig. 6. False lock probability with respect to the $C / N_{0}$. Sampling frequency at $50 \mathrm{MHz}$.

is consistent with the probability of false lock at $f_{s}=50 \mathrm{MHz}$ observed in Figure 2. The accounted $C / N_{0}$ in the Figure is in practice infinite as no noise is generate. Therefore, the figure shows the asymptotic performance of the error probability.

\section{CONCLUSION}

This paper deals with the false lock issue of high order BOC signals from an open-loop perspective. Two different strategies to estimate the time of arrival were evaluated. On the one hand, the conventional approach, which consists on finding the argument of the maximum of the correlation samples, was reviewed. On the other hand, the ML estimation of the time delay from the post-correlation samples was derived. Their asymptotic performance was assessed with respect to the sampling frequency by means of numerical simulations. The conventional estimator was observed to incur significant values of false lock probability for a large span of sampling frequency values. Only for a few sampling rates the false locking was observed to decrease substantially. These sampling rates turned out to be multiples of the subcarrier frequency. The proposed post-correlation estimator reduces the false lock probability to zero for all the sampling frequencies analyzed. Both approaches were also compared for a range of $C / N_{0}$ values. The proposed approach outperforms the standard approach and virtually removes the false locking at high $C / N_{0}$.

Future work could be devoted to the analysis of false lock in the presence of multipath propagation. The impact of false locking in the PVT solution could also be investigated.

\section{ACKNOWLEDGMENT}

This work has been funded by the European Space Agency (ESA) under project OLGNSS ('Open-loop Tracking Techniques for Snapshot-Based Positioning'), with CTTC as contractor. The views presented in the paper represent solely the opinion of the authors and not necessarily the view of ESA. Additionally, the work was partially supported by the Government of Catalonia under Grant 2014-SGR-1567.

\section{REFERENCES}

[1] N. Martin, V. Leblond, G. Guillotel, and V. Heiries, "BOC (x, y) signal acquisition techniques and performances," in ION GPS/GNSS 2003, Portland, OR, Sep., 2003, pp. 188-198.

[2] P. Fine and W. Wilson, "Tracking algorithm for gps offset carrier signals," in Proceedings of the 1999 National Technical Meeting of The Institute of Navigation, 1999, pp. 671-676.

[3] M.S. Hodgart, P.D. Blunt, and M. Unwin, "The optimal dual estimate solution for robust tracking of binary offset carrier (boc) modulation," in Proceedings of the 20th International Technical Meeting of the Satellite Division of The Institute of Navigation (ION GNSS 2007), 2001, pp. 1017-1027.

[4] M. Navarro-Gallardo, G. Seco-Granados, G. López-Risueño, and M. Crisci, "Code smoothing for boc ambiguity mitigation," in Localization and GNSS (ICL-GNSS), 2013 International Conference on. IEEE, 2013, pp. 1-6.

[5] F. M. Schubert and J. Wendel, "BOC tracking using phase and sub-carrier locked loops," in 6th European Workshop on GNSS Signals and Signal Processing, 2013.

[6] J.A. Garcia-Molina, M. Navarro-Gallardo, G. Lopez-Risueño, and M. Crisci, "Unambiguous tracking of high-order boc signals in urban environments: Channel considerations," in Satellite Navigation Technologies and European Workshop on GNSS Signals and Signal Processing (NAVITEC), 2014 7th ESA Workshop on. IEEE, 2014, pp. 1-6.

[7] J.A. Garcia-Molina, M. Navarro-Gallardo, G. Lopez-Risueño, and M. Crisci, "Robust unambiguous tracking of high-order BOC signals: A multi-correlator approach," in ION GNSS+ 2015, Tampa, FL, Sep. IEEE, 2015, pp. 1-6.

[8] A. Gusi, P. Closas, and J.A. Garcia-Molina, "False Lock Probability in BOC Signals," in ION ITM 2016, Monterey, CA, Jan., 2016.

[9] E. Kaplan and C. Hegarty, Understanding GPS: principles and applications, Artech house, 2005 